\title{
The integrated relations of fruits and vegetables with cancer
}

\begin{abstract}
Abbreviations: DNA, deoxyribonucleic acid; TNF, tumor necrosis factor; IL, interleukin; MMP, matrix metalloproteinase; COX-2, cyclooxygenase-2; uPA, urokinase plasminogen activator; ICAM-1, intercellular adhesion molecule-1; EGF, epidermal growth factor; PDGF, platelet derived growth factor; CYP1, cytochrome P450, family 1; ACF, aberrant cryptic foci; GST, glutathione s transferase; CHRP, pulp from satsuma mandarin; mRNA, messenger RNA; COX2, cyclooxygenase-2; iNOS, inducible nitric oxide synthase; Nrf2 nuclear factor (erythroid-derived 2)-like 2; QR, quinone reductase; HGPIN, high grade prostatic intraepithelial neoplasia; PSA, prostate specific antigen; POMx, pomegranate extract; HNCs, head and neck cancers; OCC, oral cavity cancer; OHPC, Oro-/Hypopharyngeal Cancer; LC, laryngeal cancer; CI, Confidence Interval; p, Probability; ESCC, esophageal squamous cell carcinoma; SRRs, summary relative risks; RR, relative risk; EPIC, European prospective investigation into cancer and nutrition
\end{abstract}

\section{Introduction}

The possibility of fruits and vegetables in reducing the risk of cancer dates back to around 40-years when the results from a small prospective study suggested that, even after allowing for the effect of smoking, people with a low intake of vitamin A from foods such as carrots and milk were at increased risk for lung cancer. ${ }^{1}$ At the same time, differences in cancer rates and diet between countries suggested that various dietary factors, including plant foods, might have important effects on cancer risk. ${ }^{2}$ Epidemiological research on fruit and vegetables and cancer has increased rapidly, and by 1992 , a meta-analysis of 156 studies have concluded that for most cancer sites, persons with low fruit and vegetable intake experience about twice the risk of cancer compared to those with a high intake, even after control for potentially confounding factors. ${ }^{3}$ Furthermore, consideration of the potential biological effects of various constituents of fruits and vegetables suggested probable mechanisms for protective effects, such as by reducing oxidative damage of DNA or increasing the activity of enzymes to detoxify the carcinogens. ${ }^{4}$

\section{Materials and methods}

The aim of this study is to find the association of consuming fruits and vegetables with the risk of different types of cancer and to brief the mechanisms involved. Fruit, vegetables, and certain components of plant foods such as fiber, have long been thought to protect against cancer. The European Prospective Investigation into Cancer and Nutrition (EPIC) is a prospective cohort that includes $>500,000$ participants from 10 European countries and has made a substantial contribution to knowledge in this research area. We incorporated the search results from the available data to summarize the published findings thus far from the EPIC study on the associations between fruit, vegetable, or fiber consumption and the risk of cancer at 8 different sites and draw the inference.

\section{Results and discussion}

It is estimated that approximately $15 \%$ of human cancers are associated with infections and inflammation. ${ }^{5}$ Various lifestyle factors,
Volume 2 Issue 5 - 2015

\author{
Dhull AK, Bansal N, Atri R, Chauhan AK, \\ KaushalV \\ Department of Radiation Oncology, Pt. B.D. Sharma Post \\ Graduate Institute of Medical Sciences Rohtak, India
}

Correspondence: Anil Kumar Dhull, Department of Radiation Oncology, Pt. B.D. Sharma Post Graduate Institute of Medical Sciences Rohtak, Haryana, India, Tel 98I20-1 I0 I , Email AnilKDhull@gmail.com

Received: March 16, 2015 | Published: June 01, 2015

such as diet, tobacco and alcohol use, environmental pollutants, radiation and infections, can cause chronic inflammation and lead to tumourigenesis. ${ }^{6}$ The markers of chronic inflammation include pro-inflammatory cytokines (i.e. tumor necrosis factor [TNF] and interleukins [ILs] $-1,-6$ and -8 ) and chemokines, pro-inflammatory enzymes 5-lipoxygenase, matrix metalloproteinase [MMP], (cyclooxygenase-2 [COX-2], and urokinase plasminogen activator [uPA]), adhesion molecules (intercellular adhesion molecule-1 [ICAM-1], vascular cell adhesion molecule-1 and endothelial leukocyte adhesion molecule-1) and certain growth factors (such as epidermal growth factor $[\mathrm{EGF}]$ and platelet-derived growth factor $[$ PDGF] $) .{ }^{7}$ Diets that include fruits, vegetables and spices may provide substantial health benefits in terms of cancer prevention and treatment by suppressing the inflammatory processes that lead to transformation, hyperproliferation and the initiation of carcinogenesis. ${ }^{6}$ Although it is not yet possible to provide quantitative estimates of the overall risks, it has been estimated that $35 \%$ of cancer deaths may be related to dietary factors. ${ }^{8}$

The types of vegetables or fruits that most often appear to be protective against cancer are raw vegetables, followed by allium vegetables, carrots, green vegetables, cruciferous vegetables, and tomatoes. The evidence for a protective effect of vegetable and fruit consumption is consistent for cancers of the stomach, esophagus, lung, oropharynx, prostate, pancreas and colon ${ }^{9}$

\section{Colorectal cancer}

Since consumption of certain dietary agents has been linked with various cancers, fruit juices have been investigated for their consistently protective effect against colon cancer. The unique biochemical composition of fruit juices is responsible for their chemo-preventive properties. ${ }^{10}$ Some studies also proved that a phytochemical-rich diet which is absorbed by the body from fruit and vegetable sources can decrease the risk of developing colon cancer. ${ }^{10}$ Fruit and vegetables are rich in dietary fibre and, although there are other important sources such as unrefined cereals, it might be expected that if dietary fibre reduces the risk of colorectal cancer, then a reduction in risk would be observed in association with high 
intakes of fruit and vegetables. ${ }^{11}$ Fruits, nuts, vegetables and grains contain major non-nutrient components called polyphenols which have chemo-preventive properties against colon cancer. The major mechanisms through which they exert this activity are through the combination of properties such as anti-proliferative, pro-apoptotic and antioxidant properties of the polyphenolics. Moreover, since fruit juices alkalize the body and provide an abundance of enzymes, vitamins, minerals, phytochemicals and other nutrients, they prove to be a better alternative for preventing the colon cancer.

\section{Pomegranate juice}

The pomegranate juice contains different types of polyphenols such as gallo, ellagitannin and flavonoid classes. It was found that ellagic acid prevents cancer initiation and inhibits the CYP1 activation of procarcinogens. ${ }^{12}$ Moreover, the ellagic acid also induces phase-2 enzymes like glutathione S-transferase. ${ }^{10}$ Boateng et al conducted a study on the effect of pomegranate juice on aberrant cryptic foci (ACF) for the purpose of analyzing the changes associated with colon cancer. ${ }^{13}$ It was established that there was a significant decrease in the number of large crypts in pomegranate juice-fed rats. This is because the activity of hepatic glutathione S transferase (GST) was found to be three times higher in the case of rats being fed with pomegranate juice. GST is well known for the scavenging of free radicals that are produced from oxidative stress and it supports the anti-oxidative mechanism of action of pomegranate in other experimental models. ${ }^{14}$

\section{Citrus juices}

Satsuma mandarin, i.e. orange is the most common citrus fruit and the pulp and juice of the citrus fruit contain flavonoids such as apigenin, naringenin, limonoids, hesperidin, nobiletin and cryptoxanthin, a carotenoid. Also, the peels of citrus fruits contain a phytochemical called tangeritin. These entire components act as chemo-preventive agents. ${ }^{10}$ The juice also contains $\beta$-cryptoxanthin, a carotenoid, and hesperidin, a flavonoid, which are potential chemo-protective compounds. $\beta$-Cryptoxanthin with non-substituted $\beta$-ionone cycles and pro-vitamin A possesses several biological activities including scavenging of free radicals, enhancement of gap junctions, immune-modulation, and regulation of enzyme activity involved in carcinogenesis and inhibition of tumorigenesis. ${ }^{15}$ Hesperidin is found in various vegetables and fruits, and is shown to exhibit antioxidant activity, anti-inflammatory effect and an inhibiting effect on prostaglandin biosynthesis. ${ }^{16} \mathrm{CHRP}$ and citrus juices also exhibit suppressing effects on hyper-cell proliferation activity induced by carcinogens in the colon, thereby inhibiting carcinogenesis. ${ }^{10}$ They also suppress mRNA expression of several cytokines [tumor necrosis factor-alpha, interleukin (IL)-1 $\beta$, IL-6] and inflammatory enzymes [COX-2 and inducible nitric oxide synthase (iNOS)] and enhance mRNA expression of $\mathrm{Nrf} 2$ in colon that received a carcinogen. $\mathrm{Nrf2}$ is a transcription factor and a key regulator of the inducible expression of enzymes such as GST and QR. Nrf2 also regulates the cytoprotective transcriptional response leading to prevention of damage to DNA, proteins and lipids, as well as recognition, repair, and removal of macromolecular damage and tissue renewal following toxicity. With cancer development in tissues there is an association of chronic inflammation regulated and mediated by cytokines. Any imbalance in their levels of production results in tumor invasion and metastasis. Inflammation is also likely to be involved with other forms of sporadic as well as heritable colorectal cancer. The positive effects of CHRP and citrus juices are attractive for reducing tumor formation when considering the relationship between inflammation and cancer development. ${ }^{17}$

\section{Prostate cancer}

Tomatoes, their related products; cruciferous vegetables and pomegranate seem to influence the risk of prostate cancer in the population and may be preventive in early prostate cancer. Tomato contains lycopene which is a powerful antioxidant and may have additional properties in DNA repair that may add to its role as a Chemopreventive agent. ${ }^{18}$ A meta-analysis of 21 epidemiological studies in 2004 observed the relation of lycopene against prostate cancer and found a weak protective effect. ${ }^{19}$ A randomized, doubleblind, placebo-controlled study of lycopene was conducted in 40-patients with evidence of HGPIN (high-grade prostatic intraepithelial neoplasia) at transurethral resection of the prostate. After one year of treatment, the rate of prostate cancer reduction was $66 \%$. Lycopene was considered an effective chemopreventive agent in the treatment of HGPIN, with no toxicity and good tolerability. ${ }^{20}$ A recent meta-analysis included eleven cohort studies and six nested case-control studies which collectively have shown the modest role of tomato in the prevention of prostate cancer. ${ }^{21}$ Further research is needed to determine the type and optimal quantity of tomato products required for prevention against prostate cancer. The other active components responsible for anti-cancer properties in prostate cancer are isothiocyanates and cruciferous vegetables including cabbage, broccoli, brussel sprouts, cauliflower and wasabi are the rich source of isothiocyanates. ${ }^{22}$ They have demonstrated induction of cell cycle arrest, inhibition of tumor invasion, angiogenesis, anti-inflammatory activity and inhibition of extracellular signal-regulated kinases in both in vitro and in vivo studies. ${ }^{18}$ Recently Bosetti et al. ${ }^{23}$ looked at 7 cohort and 6 population-based case-control studies, and observed a significantly decreased prostate cancer risk in the cruciferous vegetables intake group (relative risks $=0.90 ; 95 \%$ confidence interval $0.85-0.96)$, but not in the subgroup of cohort studies. ${ }^{23}$

\section{Pomegranate}

Its juice contains high concentrations of both tannin and flavonoid anti-oxidants. ${ }^{1}$ A large multi-centric study performed in 2004 looked at the effect of three preparations of pomegranate supplements on three prostate cancer cell lines and on a xenograft model. This found significant anti-tumor properties of pomegranate mediated by the induction of apoptosis and changes in cell cycle. ${ }^{24} \mathrm{~A}$ role for pomegranate polyphenols in the inhibition of gene expression in androgen receptors in advanced prostate cancer cell models has also been demonstrated. ${ }^{25}$ In a phase II study on 46 prostate cancer patients who had raised PSA levels were given 8 ounces of pomegranate juice daily and serial PSA levels were taken. The observations were promising with a significant prolongation of the PSA doubling time from a mean of 15 months to 54 months, along with a prolongation of disease stabilization. ${ }^{26}$ Another recent randomized study looked at 70 men awaiting radical prostatectomy who were either taking pomegranate extract (POMx) or placebo tablets daily, for up to four weeks before surgery. At the end of the study, there were no significant differences in the clinical or pathological features of prostate cancer in either group. ${ }^{27}$ Though, greater accumulation of pomegranate metabolites within prostate tissue might confer a protective benefit against oxidative DNA damage. Thus, pomegranate supplementation may have a role in both prevention and delaying progression of prostate cancer, but available data are conflicting and the mechanisms involved are idiopathic. ${ }^{18}$ There has been much interest in the possibility that fruits and vegetables, such as tomatoes, which are rich in the carotenoid lycopene, might reduce the risk for prostate cancer. Studies of soybeans and prostate cancer have suggested that this may also help to reduce risk, but the results are not conclusive. ${ }^{28}$ 


\section{Head \& neck cancer}

The association of fruits and vegetables with cancer risk has been investigated in several case-control studies, which have observed an approximate $50 \%$ reduction in the risk of head and neck cancers (HNCs) in people with high intakes of fruit and vegetables. ${ }^{11}$ There is limited prospective data on the relationship between consumption of vegetables and fruits and the risk of head \& neck cancer (HNC) subtypes [i.e., oral cavity cancer (OCC), oropharyngeal/ hypopharyngeal cancer (OHPC) and laryngeal cancer (LC)]. Total vegetable and fruit consumption is inversely associated with risk of $\mathrm{HNC}$ overall [multivariable-adjusted rate ratios for highest vs. lowest quartile, $0.61,95 \%$ confidence interval (CI) $0.44-0.85$, p trend 0.002 ] and all HNC subtypes, with the strongest associations for OCC. ${ }^{29}$ Freedman et al., ${ }^{30}$ reported that, compared with people who consumed $\square 1.5$ portions of fruit and vegetables each day, people with intakes of $\square 5.8$ portions per day had a relative risk of $0.71 .{ }^{30}$ In conclusion, consumption of vegetables and fruits may protect against $\mathrm{HNC}$ and its subtypes.

\section{Esophageal cancer}

The possible role of fruit and vegetables has been investigated in several case control studies, which on average have observed that people with relatively high intakes of fruit and vegetables have a $40-50 \%$ lower risk of total esophageal cancer than people with low intakes of fruit and vegetables. ${ }^{11}$ Quantification of the association between the intake of fruit and vegetables and risk of esophageal squamous cell carcinoma (ESCC) is controversial even though several studies have explored this association. ${ }^{31}$ The few prospective data available suggest that intake of fruit and vegetable is inversely associated with the risk for squamous cell carcinoma, but not with the risk for adenocarcinoma. Therefore, the data is consistent with the hypothesis that adequate intakes of fruit and vegetables reduce the risk for squamous cell carcinoma of the esophagus, but it is still possible that the association seen is due to residual confounding by smoking and alcohol. ${ }^{11}$ In a meta-analysis of 32 studies involving 10,037 cases of ESCC, random-effects models were used to calculate summary relative risks (SRRs) and the corresponding 95\% confidence intervals (CIs). The SRRs for the highest vs. lowest intake were $0.56(95 \%$ CI, 0.45-0.69) for vegetable intake and 0.53 (95\% CI, 0.44-0.64) for fruit intake. Similar results were observed in a linear dose-response analysis. A non-linear association for intakes of fruit and vegetables was evident from the study. These statistics support the hypothesis that intakes of vegetables and fruit may significantly reduce the risk of ESCC. ${ }^{31}$

\section{Stomach cancer}

There is a longstanding hypothesis that risk may be reduced by adequate intakes of fruit and vegetables, perhaps due to their content of antioxidant nutrients such as ascorbic acid. Data from case-control studies support this hypothesis, but the results from prospective studies have mostly shown weak or null associations. ${ }^{11}$ In a metaanalysis by Wang et al., ${ }^{31}$ a total of 24 studies were analyzed. There were $>2.4$ million individuals (6632 gastric cancer events) with a median follow-up of 10-years. Based on the high versus low analysis, consumption of fruit, but not vegetables, may reduce risk of gastric cancer (fruit, SRR $=0.90,95 \%$ CI, 0.83-0.98; vegetable, SRR $=0.96$, 95\% CI, 0.88-1.06). The same results were also shown in the linear dose-response analysis (per $100 \mathrm{gm} /$ day) (fruit, SRR $=0.95,95 \% \mathrm{CI}$, 0.91-0.99; vegetable, $\mathrm{SRR}=0.96,95 \%$ CI, 0.91-1.01). Significant inverse associations emerged in non-linear models for consumption of fruit, but not for consumption of vegetables. Findings from this metaanalysis indicate a significant protective effect for the consumption of fruit on gastric cancer risk, but not for the consumption of vegetables. ${ }^{2}$

\section{Lung cancer}

Many observational studies have found that lung cancer patients report a somewhat lower intake of fruits and vegetables than controls, but the effect of smoking is so large, compared with the small association with diet, that residual confounding by smoking is likely, and recent large prospective analyses with detailed adjustment for smoking have not shown a convincing association between fruit and vegetable intake and the risk for lung cancer. ${ }^{11}$

\section{Breast cancer}

Several large prospective studies have investigated whether high intakes of fruit and vegetables might be associated with a reduced risk of breast cancer, but no fruitful results were observed.$^{33}$ In the Women's Health Initiative randomized trial, an increase in fruit and vegetable intake of 1.1 servings per day (combined with an increase in grain intake and a reduction in fat intake) did not cause a significant change in the incidence of breast cancer after 8 -years. ${ }^{34}$ It seems unlikely that high intakes of fruit and vegetables in general have a significant protective effect, but it is still possible that specific vegetables rich in isoflavones, especially soybeans, might have a protective effect by reducing the estrogenic stimulation of the breast cells. ${ }^{33}$

\section{Pancreatic cancer}

Most of the nutrients contained in fruits and vegetables and their supplements are associated with a reduced risk of pancreatic cancer. Some results have shown a significant trend ( $p$-value $<0.05$ ), inverse association between pancreatic cancer and nutrient/supplement groupings in a dose-dependent manner including selenium, magnesium, potassium, alpha-carotene, beta-carotene, beta-cryptoxanthin, lutein, zeaxanthin, niacin, total alpha-tocopherol, pyridoxin, total retinoic acid activity and ascorbic acid. ${ }^{35}$ Vegetable consumption (RR, 0.71; $95 \% \mathrm{CI}, 0.57-0.88$ ) and fruit consumption (RR, 0.73; 95\% CI, 0.60$0.90)$ provided the greatest degree of protection against pancreatic diseases on the basis of meta-analyses. Vegetable consumption had stronger association with protection against acute pancreatitis and fruit consumption with protection against pancreatic cancer. ${ }^{36}$

\section{Conclusion}

The risk of cancers of the upper gastrointestinal tract was inversely associated with fruit intake but was not associated with vegetable intake. The risk of colorectal cancer was inversely associated with intakes of total fruit and vegetables and total fiber, and the risk of liver cancer was also inversely associated with the intake of total fiber. The risk of cancer of the lung was inversely associated with fruit intake but was not associated with vegetable intake; this association with fruit intake was restricted to smokers and might be influenced by residual confounding due to smoking. There was a borderline inverse association of fiber intake with breast cancer risk. For the other cancer sites studied (stomach, pancreas and prostate) there were no reported significant associations of risk with intakes of fruit, vegetables, or fiber. ${ }^{37}$

Table 1 is showing some of the studies which have recently reported the association of fruit and vegetables with the risk of different types of cancer. These results provide some indication of public health impact, but need to be interpreted cautiously because they may be influenced by confounding factors. 
Table I Association of intake of fruits and vegetables to cancer risk

\begin{tabular}{|c|c|c|c|c|}
\hline Author & Study design & Site of cancer & Diet & Association \\
\hline \multirow[t]{5}{*}{ Bradbury et al. ${ }^{37}$} & Cohort study, & Upper GI Cancer & Fruits & Inversely associated \\
\hline & 2014 & & Vegetables & Not associated \\
\hline & & Colorectal & Fruits \& Vegetables & Inversely associated \\
\hline & & Lung & Fruits & Inversely associated \\
\hline & & & Vegetables & Not associated \\
\hline \multirow[t]{2}{*}{ Wang et al. ${ }^{32}$} & Meta-analysis, 2014 & Stomach & Fruits & Inversely associated \\
\hline & & & Vegetables & Not associated \\
\hline \multirow[t]{2}{*}{ Liu et al. ${ }^{31}$} & Meta-analysis, 2013 & Esophagus & Fruits \& Vegetables & Inversely associated \\
\hline & & SCC & & \\
\hline \multirow[t]{2}{*}{ Maasland. et al. ${ }^{29}$} & Cohort study, & Head \& Neck Cancer & Fruits \& Vegetables & Inversely associated \\
\hline & 2014 & & & \\
\hline \multirow[t]{2}{*}{ Alsamarrai et al. ${ }^{36}$} & Cohort study, & Pancreatic Cancer & Fruit & Inversely associated \\
\hline & 2014 & & & \\
\hline
\end{tabular}

\section{Acknowledgements}

None.

\section{Conflicts of interest}

The author declares that there is no conflict of interest.

\section{Funding}

None.

\section{References}

1. Bjelke E. Dietary vitamin A and human lung cancer. Int $J$ Cancer. 1975;15(4):561-565.

2. Armstrong B, Doll R. Environmental factors and cancer incidence and mortality in different countries, with special reference to dietary practices. Int J Cancer. 1975;15(4):617-631.

3. Block G, Patterson B, Subar A. Fruit, vegetables, and cancer prevention:a review of the epidemiological evidence. Nutr Cancer. 1992;18(1):1-29.

4. Steinmetz KA, Potter JD. Vegetables, fruit, and cancer. II Mechanisms. Cancer Causes Control. 1991;2(6):427-442.

5. Coussens LM, Werb Z. Inflammation and cancer. Nature. 2002;420(6917):860-867.

6. Sung B, Prasad S, Yadav VR, et al. Cancer and diet:How are they related? Free Radic Res. 2011;45(8):864-879.

7. Aggarwal BB, Shishodia S, Sandur SK, et al. Inflammation and cancer:how hot is the link? BiochemPharmacol. 2006;72(11):16051621 .

8. Doll R, Peto R. The causes of cancer: quantitative estimates of avoidable risks of cancer in the United States today. J Natl Cancer Inst. 1981;66(6):1191-1308

9. Steinmetz KA, Potter JD. Vegetables, fruit, and cancer prevention: a review. J Am Diet Assoc. 1996;96(10):1027-1039.

10. Jaganathan SK, Vellayappan MV, Narasimhan G, et al. Role of pomegranate and citrus fruit juices in colon cancer prevention. World $J$ Gastroenterol. 2014;20(16):4618-4625.
11. Key TJ. Fruit and vegetables and cancer risk. Br JCancer. 2011;104(1):611.

12. Barch DH, Rundhaugen LM, Stoner GD, et al. Structure-function relationships of the dietary anticarcinogenellagic acid. Carcinogenesis. 1996;17(2):265-269.

13. Boateng J, Verghese M, Shackelford L, et al. Selected fruits reduce azoxymethane (AOM)-induced aberrant crypt foci (ACF) in Fisher 344 male rats. Food Chem Toxicol. 2007;45(5):725-732.

14. Rosenblat M, Hayek T, Aviram M. Anti-oxidative effects of pomegranate juice (PJ) consumption by diabetic patients on serum and on macrophages. Atherosclerosis. 2006;187(2):363-371.

15. Tanaka $\mathrm{T}$, Sugiura $\mathrm{H}$, Inaba $\mathrm{R}$, et al. Immunomodulatory action of citrus auraptene on macrophage functions and cytokine production of lymphocytes in female BALB/c mice. Carcinogenesis. 1999;20(8):14711476.

16. Kelloff GJ, Boone CW, Crowell JA, et al. New agents for cancer chemoprevention. J Cell BiochemSuppl. 1996;26:1-28.

17. Surh YJ. NF-kappa B and Nrf2 as potential chemopreventive targets of some anti-inflammatory and antioxidative phytonutrients with anti-inflammatory and antioxidative activities. Asia Pac J ClinNutr 2008;17(Suppl 1):269-272.

18. Mandair D, Rossi RE, Pericleous M, et al. Prostate cancer and the influence of dietary factors and supplements:a systematic review. Nutr Metab (Lond.). 2014;11:30.

19. Etminan M, Takkouche B, Caamano-Isorna F. The role of tomato products and lycopene in the prevention of prostate cancer:a metaanalysis of observational studies. Cancer Epidemiol Biomarkers Prev. 2004;13(3):340-345.

20. Mohanty NK, Saxena S, Singh UP, et al. Lycopene as a chemopreventive agent in the treatment of high-grade prostate intraepithelial neoplasia. UrolOncol. 2005;23(6):383-385.

21. Chen J, Song Y, Zhang L. Lycopene/Tomato consumption and the risk of prostate cancer: a systematic review and meta-analysis of prospective studies. J Nutr Sci Vitaminol (Tokyo). 2013;59(3):213-223.

22. Liu RH. Potential synergy of phytochemicals in cancer prevention: mechanism of action. J Nutr. 2004;134(12 Suppl):3479S-3485S. 
23. Bosetti C, Filomeno M, Riso P, et al. Cruciferous vegetables and cancer risk in a network of case-control studies. Ann Oncol. 2012;23(8):21982203.

24. Albrecht M, Jiang W, Kumi-Diaka J, et al. Pomegranate extracts potently suppress proliferation, xenograft growth, and invasion of human prostate cancer cells. J Med Food. 2004;7(3):274-283.

25. Hong MY, Seeram NP, Heber D. Pomegranate polyphenols downregulate expression of androgen-synthesizing genes in human prostate cancer cells overexpressing the androgen receptor. J NutrBiochem. 2008;19(12):848-855.

26. Pantuck AJ, Leppert JT, Zomorodian N, et al. Phase II study of pomegranate juice for men with rising prostate-specific antigen following surgery or radiation for prostate cancer. Clin Cancer Res. 2006;12(13):4018-4026.

27. Freedland SJ, Carducci M, Kroeger N, et al. A double-blind, randomized, neoadjuvant study of the tissue effects of POMx pills in men with prostate cancer before radical prostatectomy. Cancer Prev Res. 2013;6(10):1120-1127.

28. Hwang YW, Kim SY, Jee SH, et al. Soy food consumption and risk of prostate cancer: a meta-analysis of observational studies. Nutr Cancer. 2009;61(5):598-606.

29. Maasland DH, Van den Brandt PA, Kremer B, et al. Consumption of vegetables and fruits and risk of subtypes of head-neck cancer in the Netherlands Cohort Study. Int J Cancer. 2015;136(5):E396-E409.

30. Freedman ND, Park Y, Subar AF, et al. Fruit and vegetable intake and head and neck cancer risk in a large United States prospective cohort study. Int J Cancer. 2008;122(10):2330-2336.
31. Liu J, Wang J, Leng Y, Lv C. Intake of fruit and vegetables and risk of esophageal squamous cell carcinoma: a meta-analysis of observational studies. Int J Cancer. 2013;133(2):473-485.

32. Wang Q, Chen Y, Wang X, et al. Consumption of fruit, but not vegetables, may reduce risk of gastric cancer: results from a metaanalysis of cohort studies. Eur J Cancer. 2014;50(8):1498-1509.

33. Michels KB, Mohllajee AP, Roset-Bahmanyar E, et al. Diet and breast cancer: a review of the prospective observational studies. Cancer. 2007;109(12 Suppl):2712-2749.

34. Prentice RL, Caan B, Chlebowski RT, et al. Low-fat dietary pattern and risk of invasive breast cancer: the Women's Health Initiative Randomized Controlled Dietary Modification Trial. JAMA. 2006;295(6):629-642.

35. Jansen RJ, Robinson DP, Stolzenberg-Solomon RZ, et al. Nutrients from Fruit and Vegetable Consumption Reduce the Risk of Pancreatic Cancer. J Gastrointest Cancer. 2013;44(2):152-161.

36. Alsamarrai A, Das SL, Windsor JA, et al. Factors That Affect Risk for Pancreatic Disease in the General Population: A Systematic Review and Meta-analysis of Prospective Cohort Studies. Clin GastroenterolHepatol. 2014;12(10):1635-1644.

37. Bradbury KE, Appleby PN, Key TJ. Fruit, vegetable, and fiber intake in relation to cancer risk: findings from the European Prospective Investigation into Cancer and Nutrition (EPIC). Am J Clin Nutr. 2014;(100 Suppl 1):394S-398S. 